\title{
Tumor immune microenvironment: sanctuary of tumor and target for immunotherapy
}

\author{
Xuan Gu, Qi Tang** \\ Key Lab of Antibody Technique of National Health Commission, Nanjing Medical University, Nanjing, Jiangsu, 211166, China
}

\begin{abstract}
The tumor immune microenvironment (TIME) is the cellular environment in which tumors exist. This includes: surrounding blood vessels, immune cells, fibroblasts, bone marrow-derived inflammatory cells, lymphocytes, signaling molecules, immune checkpoint proteins and the extracellular matrix (ECM). The TIME plays a critical role in cancer progression and regulation. Tumors can influence the microenvironment by releasing extracellular signals, promoting tumor angiogenesis and inducing peripheral immune tolerance, while the immune cells in the microenvironment can affect the growth and evolution of cancerous cells. The molecules and cells in the TIME influence disease outcome by altering the balance of suppressive versus cytotoxic responses in the vicinity of the tumor. Having a better understanding of the tumor immune microenvironment will pave the way for identifying new targets for immunotherapies that promote cancer elimination.
\end{abstract}

Keywords: tumor immune microenvironment, immune checkpoints, tumor metastasis, cancer immunotherapy

\section{INTRODUCTION}

An established tumor is a complex tissue, comprising not only of tumor cells but also stromal cells, vasculature, extracellular matrices (ECM) and immune cells ${ }^{[1]}$, which can either promote or hinder tumor growth, progression and metastasis. The cross-talk between immune cells and tumor cells has hampered progress in understanding the mechanisms of immunosuppression by which tumor cells circumvent immune responses. Immunoescape is one of the cancer hallmarks that have been proposed in the last decade, which provide a solid foundation for understanding the biology of cancer ${ }^{[2]}$. Inflammation in the development of cancers has been implicated since the seminal observation made by Virchow in as early as 1863 , in which chronic inflammation was shown to

*Correspondence to: Qi Tang, PhD. Key Lab of Antibody Technique of National Health Commission, Nanjing Medical University, Nanjing, Jiangsu, 211166, China. E-mail: qitang@njmu.edu.cn.

Conflict of interests: The authors declared no conflict of interests. create a microenvironment conducive to tumor genesis $^{[3]}$. This microenvironment is named the tumor immune microenvironment (TIME), and has recently raised much interest. The communication between tumor cells and the TIME is a dynamic process. When the anti-tumor immune response is strong, the cancer stays under control. However, multiple elements of suppression play important roles in the TIME, including immune cells such as $\mathrm{T}$ regulatory cells, myeloid suppressive cells, tumor-associated macrophages, and mast cells, and molecules such as checkpoints that control $\mathrm{T}$-cell differentiation (for example, CTLA-4) and effector functions (such as PD-1 $)^{[4]}$. A pharmacological blockade of these inhibitory elements can tip the balance toward anticancer effector $\mathrm{T}$ cells ${ }^{[5]}$. Thus, a better understanding of TIME, including classes of TIME, immune cells and immune checkpoints, relationships between tumor genotypes and TIME, as well as the function of TIME in tumor metastasis may benefit the efficiency of cancer immunotherapy. 


\section{CLASSIFICATION OF THE TIME}

Checkpoint blockade therapy has been proven to be active in many cancer types, but emerging evidence shows it limits the therapeutic benefit to a subset of patients in each cancer entity ${ }^{[6]}$. It is necessary for us to classify the TIME to make a better understanding about why some patients show no response to checkpoint blockade therapy. The TIME can be characterized into three classes.

Infiltrated-excluded (I-E) TIME is the TIME populated with immune cells, but relatively void of cytotoxic lymphocytes (CTLs) in the tumor core ${ }^{[7]}$. I-E TIME is associated with various epithelial cancers, in which tumor-associated macrophages (TAMs) along the tumor margin are able to prevent CTLs from entering the tumor core $^{[8]}$. I-E TIME contains CTLs with low expression of the activation-marker GZMB (GRZB) and IFNG and poor infiltration of CTLs into the tumor core $^{[7]}$. The lack of activation-marker expression and exclusion from the tumor core shows immunological ignorance, in which state adaptive immunity cannot recognize or respond to a pathogen or malignancy ${ }^{[7]}$. I-E TIME lacks not only $\mathrm{T}$ cells but also the up-regulation of immune inhibitory mechanisms ${ }^{[9]}$. Therefore, it is not surprising that checkpoint blockade is not effective against this subgroup of patients ${ }^{[10]}$.

Infiltrated-inflamed (I-I) TIME is characterized by a high infiltration of CTLs expressing PD-1 and leukocytes and tumor cells expressing the immune-dampening PD-1 ligand (PD-L1) ${ }^{[8]}$. Immune escape mechanisms that allow the co-existence of an anti-tumor immune response and the tumor itself in I-I TIME have been reported in several papers ${ }^{[9-11]}$. CTLs in I-I TIME are related to the up-regulation of immune inhibitory mechanisms which can eliminate immunogenic tumor cells and leave only non-immunogenic tumor cells. This process displays a potent form of immune escape. Immune suppression in I-I TIME is being harnessed to activate the endogenous $\mathrm{T}$-cell response to the blocking of immune inhibitory mechanisms ${ }^{[12]}$.

A subclass of I-I TIME, termed TLS-TIME, contains tertiary lymphoid structures (TLSs), including and activated conventional $\mathrm{T}$ cells, regulatory $\mathrm{T}$ cells, B cells and dendritic cells (DCs) ${ }^{[13]}$. TLSs also act as sites of lymphoid recruitment and immune activation in the settings of enhanced inflammation ${ }^{[13]}$. TLSs allow $\mathrm{T}$ cells to differentiate into effector cells upon contacting with mature DCs and B cells ${ }^{[14]}$. Thus, TLS represents sites for anti-tumor responses, which confer long-term protection against metastasis and correlate with a good prognosis for the patients. In conclusion, the classifications of the TIME can improve the understanding of how the different kinds of tumor immune microenvironment affect the anti-tumor responses or even the immune escape responses.

\section{IMIMUNE CELLS OF THE TIME}

Tumors are organized tissues with numerous reciprocal local and systemic connections with immune cells including T lymphocytes, B lymphocytes, as well as myeloid cells including macrophages, myeloid-derived suppressor cells, DCs, and mast cells ${ }^{[15]}$. These comprise the tumor immune microenvironment (Fig. 1).

Many tumors express antigens that can be recognized

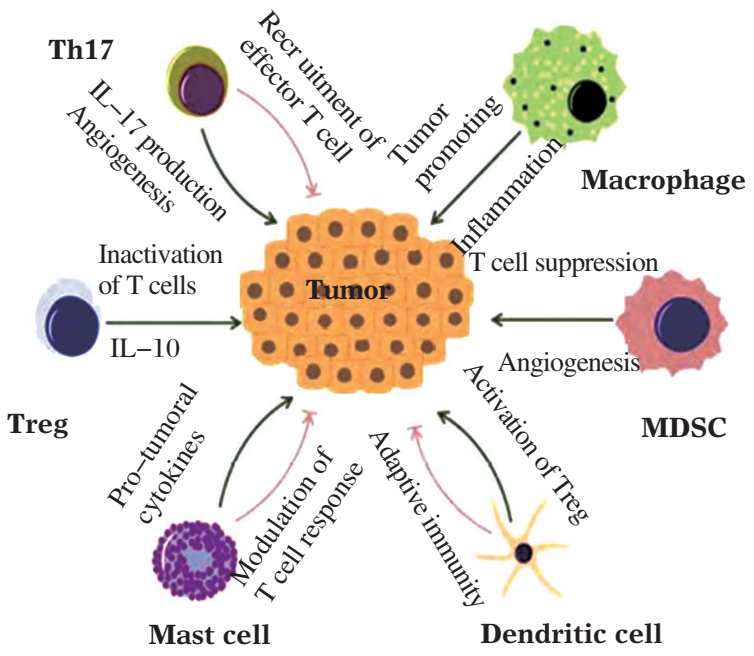

Fig. 1 Different roles of immune cells in the TIME. Th17 cells are able to eliminate cancer cells by the recruitment of $\mathrm{CD}^{+}$effector T cells, and can also stimulate angiogenesis by the production of IL-17. Tregs can inactivate T cell-mediated tumor immunity by the secretion of IL-10. Tumor-associated macrophages (TAMs) can facilitate the tumor-promoting inflammation by the secretion of a large amount of cytokines. Myeloid derived suppressor cells (MDSCs) can decrease the activity of antigen-presenting cells (APCs), natural killer (NK) and T cells, and also induce angiogenesis in the hypoxic microenvironment. DCs participate as APCs that can promote adaptive immunity. However, DCs can favor tumor progression with mediating tolerance in certain circumstances. Similarly, mast cells, another type of APCs, have also been identified to modulate anti-tumor immunity, while can generate a series of pro-angiogenic factors to promote tumor progression. (Courtesy of Dr. Song Nan, et al.)

by $\mathrm{T}$ lymphocytes, suggesting that $\mathrm{T}$ cell-mediated immunity may be harnessed for the immunotherapy of cancers ${ }^{[16]}$. Within the TIME, many $\mathrm{T}$ cells have been observed, especially at the invasive tumor margin and in draining lymphoid organs. Among these, $\mathrm{CD}^{+}$memory $\mathrm{T}$ cells and $\mathrm{CD} 4^{+} \mathrm{T}$ helper $1(\mathrm{Thl})$ cells function as the major anti-tumor immune effector cells, which secret many cytokines that can prevent and suppress the development of cancers ${ }^{[17]}$. $\mathrm{CD}^{+}$effector $\mathrm{T}$ cells can recognize endogenous peptides displayed on the surface 
of tumor cells within the groove of HLA class I molecules. After $\mathrm{CD}^{+} \mathrm{T}$ cells recognize tumor specific peptides, they induce the killing of target tumor cells, or promote tumor destruction via the secretion of effector cytokines such as interferon- $\gamma(\mathrm{IFN}-\gamma)$ and $\mathrm{TNF}^{[18]}$. After activation, naïve $\mathrm{CD} 4^{+} \mathrm{T}$ cells will differentiate into various subsets of $\mathrm{T}$ helper cells, including Thl, Th2, and Th17 cells ${ }^{[19]}$. Thl cells produce IFN- $\gamma$ and function as immunity against intracellular pathogens. Th2 cells are able to assist B cells for the production of antibodies. Th17 cell can either suppress tumor cells by promoting the activation of tumor-specific $\mathrm{CD}^{+} \mathrm{T}$ cells and recruiting of DCs into TIME or exhibit protumor functions of certain cytokines ${ }^{[20]}$. Regulatory $\mathrm{T}$ cells (Tregs) inhibit the expression of co-stimulatory molecules on the surface of DCs, thus inactivate T cells ${ }^{[21]}$. Tregs also suppresses Th $17^{[22]}, \mathrm{CD}^{+} \mathrm{T}$ cells ${ }^{[23]}$ and $\mathrm{B}$ cells activity ${ }^{[24]}$ in different ways. B cells in the tumor immune microenvironment can promote disease progression by secreting cytokines.

Studies suggest that among myeloid lineage cells, myeloid derived suppressive cells (MDSCs), mast cells, and most TAMs promote tumor development in the TIME ${ }^{[25]}$. Human MDSCs can be divided into three subsets, M-MDSCs, PMN-MDSCs and LinHLA-DR-CD $33^{+}$MDSCs ${ }^{[26]}$. PMN-MDSCs suppress immune responses by producing TGF- $\beta$ or inducing nitration of $\mathrm{T}$-cell receptors (TCR), which makes $\mathrm{T}$ cells unresponsive to antigen stimulation. M-MDSCs can differentiate into TAMs, which promote T cell apoptosis and lead to immune suppression ${ }^{[27]}$. MDSCs produce large numbers of MMPs, which has a profound impact on tumor progression and metastasis through modulation of tumor angiogenesis and tumor cell inva$\operatorname{sion}^{[28]}$.

Macrophages differentiate into two major subsets, including the pro-inflammatory Ml-type and antiinflammatory M2-type. M1-type macrophages are activated by IFN- $\gamma$ and lipopolysaccharide and are characterized by their high expression of IL-12 and low expression of IL-10. This cytokine profile promotes the development of a type $1 \mathrm{~T}$ cell response which facilitates anti-tumor immunity. The TIME can polarize macrophages towards an M2-type, giving rise to $\mathrm{TAMs}^{[29]}$. M2-type macrophages are activated by IL4, IL-13, IL-10 and glucocorticoid, which can produce high levels of IL-10 and low levels of IL-12 and promote tumor progression ${ }^{[30,31]}$.

The major functions of DCs are to process and present antigen for the $\mathrm{CD} 4^{+}$and $\mathrm{CD} 8^{+} \mathrm{T}$ cells. DCs in the TIME act as effective antigen-presenting cells (APCs) inducing specific anti-tumor immune responses ${ }^{[32]}$.
However, DCs often have impaired functions even if they recognize tumor antigen, because DCs may remain immature or may gain the expression of various immunosuppressive molecules. Multiple conditions and factors within the TIME can cause DC abnormalities ${ }^{[32]}$. The number of DCs often decreases due to tumor-induced immunosuppression ${ }^{[33]}$. In recent years, DCs presenting tumor-specific antigens are being developed as vaccines to induce immune responses to regress tumors and prevent relapse.

Mast cells (MCs) infiltrating in the TIME have been found related to tumor development, meaning that MC has become a novel prognostic marker. Mast cells can induce the release of pro-angiogenic factors such as Ang-1 and VEGF-A ${ }^{[34]}$. Mast cells are also APCs, interact with both $\mathrm{T}$ cells and $\mathrm{B}$ cells, and modulate immune responses. Mast cells can recruit eosinophils, neutrophils, and activate anti-tumor adaptive $\mathrm{T}$ and $\mathrm{B}$ cell responses. However, the recruitment of Tregs into the tumor microenvironment is mediated by mast cellderived adenosine ${ }^{[33]}$. Thus, mast cells play a role in either promoting angiogenesis, or regulating the antitumor immunity. Investigating the role of immune cells in the TIME may provide a gateway to the development of innovative and effective strategies for cancer immunotherapy ${ }^{[35]}$.

\section{IMIMUNE CHECKPOINTS OF THE TIME}

Immune checkpoints are important immune regulators in maintaining immune homeostasis and preventing autoimmunity ${ }^{[36]}$. Under normal circumstances, immune checkpoints allow the immune system to respond to infection and malignancy while protecting tissues from any harm that may derive from the condition. However, some of these immune-checkpoint proteins expressed on malignant cells may deregulate antitumor immunity and favor tumor growth ${ }^{[37]}$. So far, many immune checkpoint molecules have been studied, such as PD-1 ${ }^{[38]}$, PD-L1/2, cytotoxic T lymphocyte antigen-4 $(\mathrm{CTLA}-4)^{[39]}$, lymphocyte-activation gene 3(LAG$3)^{[40]}$, T-cell immunoglobulin and mucin-domaincontaining molecule-3 (TIM-3), B and T lymphocyte attenuator (BTLA) ${ }^{[41]}$ and so on (Fig. 2).

Present immune checkpoint molecules research is mainly focusing on CTLA-4, PD-1 and its ligands PD-L1 (B7H1) and PD-L2 (B7-DC). CTLA-4 is thought to play a role in regulating the magnitude of early $\mathrm{T}$ cell activation, while PD-1 is expressed upon $\mathrm{T}$ cell activation and regulates effector $\mathrm{T}$ cell activity $^{[41]}$, and restricts CTLA-4 expression only to T cells; while PD-1 is found on T cells, B cells and NK cells. CTLA-4 is a cell-surface receptor expressed by acti- 
vated $\mathrm{T}$ cells with homology to the $\mathrm{T}$ cell costimulatory molecule CD28. Although CD28 and CTLA-4 are both ligands for B7-1 (CD80) and B7-2 (CD86), they serve opposing roles in regulating $\mathrm{T}$ cell activation ${ }^{[4]}$. CD28 provides costimulatory signals required for $\mathrm{T}$ cell activation while CTLA-4 modulates T cell responses by raising the activation threshold for $\mathrm{T}$ cell priming ${ }^{[42]}$.

In response to immune attack, cancer cells overexpress PD-L1. They bind to the PD-1 receptor on $\mathrm{T}$ cells, inhibiting the activation of $\mathrm{T}-$ cells, thus suppressing $\mathrm{T}$-cell attack and inducing tumor immune escape $^{[18]}$. Tumor cells form a suitable tumor immune microenvironment and continue to proliferate. The PD-1/PD-Ll pathway regulates immune suppression by multiple mechanisms, specifically the performance of: (1) CD28, which sustains T cell survival by a driving expression of the anti-apoptotic gene $\mathrm{Bcl}-\mathrm{xL}$. PD-1 prevents $\mathrm{Bcl}-\mathrm{xL}$ expression by inhibiting PI3K activation (which is essential for upregulation of Bcl$\mathrm{xL})$. PD-1 induces apoptosis of activated T cells ${ }^{[43]}$. (2) PD-Ll is able to promote the generation of induced Tregs by down-regulating the mTOR, AKT, S6 and the phosphorylation of ERK2 and increasing PTEN, thus restraining the activity of the effector T-cell ${ }^{[44]}$. (3) PD-1 inhibits the proliferation of T cells by preventing the phosphorylation of PKC-theta, which is essential for IL-2 production, and arresting $\mathrm{T}$ cells in the Gl phase ${ }^{[45]}$. (4) The exhaustion of tumor infiltrating lymphocytes (TILs) in the TIME is closely related to the PD-Ll expression of tumor cells or myeloid cells derived from tumor. (5) PD-1/PD-L1 blocks the downstream signaling events triggered by $\mathrm{Ag} / \mathrm{MHC}$ engagement of the TCR and co-stimulation through CD28, resulting in impaired $\mathrm{T}$ cell activation and $\mathrm{IL}-2$ production ${ }^{[18]}$.

There are also some newly emerging immune checkpoints, such as LAG-3, BTLA, and TIM-3.

LAG-3 is a single transmembrane protein with the three-Ig extracellular domains, which expresses on activated T cells, regulatory T cells, NK cells, DCs, and B cells ${ }^{[40]}$. LAG-3 acts to inhibit the immune response by inhibiting effector $\mathrm{T}$ cell killing, and through Treg cellmediated immune suppression.

BTLA is an immunoglobulin domain-containing glycoprotein expressed on T cells, resting B cells, macrophages, and DCs ${ }^{[4]}$. BTLA acts as an inhibitory receptor on T cells, and anti-BTLA treatment can cause $\mathrm{T}$ cell proliferation ${ }^{[46]}$. TIM-3 is upregulated in various types of cancer, which can inhibit Thl cell responses ${ }^{[47]}$.

\section{TUMOR GENOTYPES AND THE TIME}

To have a better understanding of the tumor developing mechanisms, we have to figure out how tumor on-

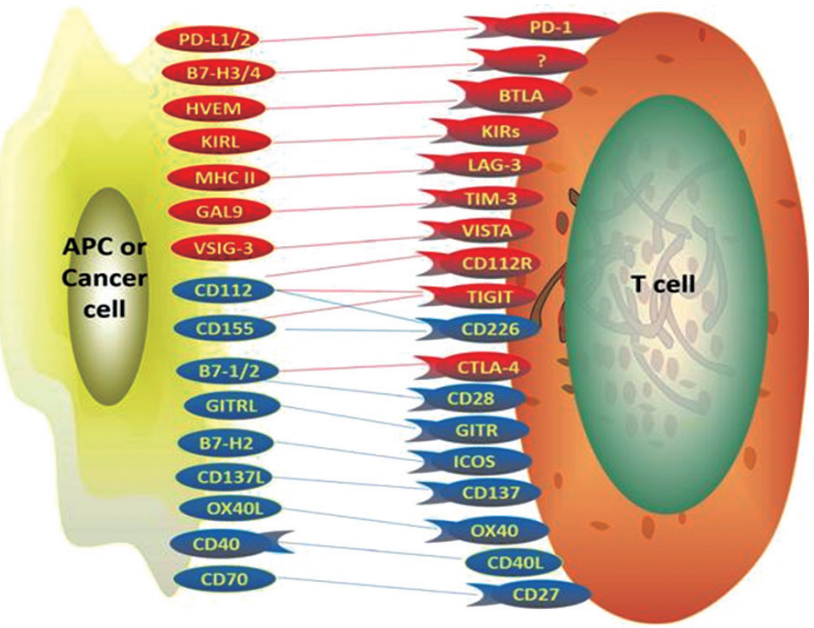

Fig. 2 Stimulatory and inhibitory immune checkpoints. The blockade of inhibitory immune checkpoints can regulate $\mathrm{T}$-cell activation and prevent immune escape of cancer cells within the tumor immune microenvironment. The activation of stimulatory immune checkpoints can augment the effect of immune checkpoint inhibitors in cancer therapeutics. Red, inhibitory immune checkpoints; blue, stimulatory immune checkpoints.(Courtesy of Dr. Feng Xu, et al.)

cogenes and mutation landscapes determine the composition of the TIME. There are several examples showing relationships between tumor genotypes and the TIME.

Deficient DNA mismatch repair (MMR) results in a strong mutate phenotype known as microsatellite instability (MSI), which is characterized by length alterations within simple repeated sequences ${ }^{[48]}$. In MSI cancers, MMR deficiency generates many aberrant proteins truncated by frameshift mutations. Inactivating mutations in the HLA and $\beta 2-$ microglobulin genes are often observed in MSI cancers, by which cancer cells may escape immune recognition by CTLs. Analyses of tumor genotypes have revealed that most tumors within the Thl/CTL immune microenvironment have defects in mismatch repair, as evidenced by MSI ${ }^{[48]}$. To counterbalance this active Thl/CTL immune microenvironment, MSI cancers selectively showed highly upregulated expression of multiple immune checkpoint proteins, such as PD-1, PD-Ll, CTLA-4, and $\mathrm{LAG}-3^{[37]}$. These findings link tumor genotype with the immune microenvironment and explain why MSI tumors are not naturally eradicated, despite the active Thl/CTL microenvironment.

$\mathrm{BRAF}^{\mathrm{V} 600 \mathrm{E}}$ is a mutated and oncogenic form of the MAPK family member BRAF in melanoma ${ }^{[49]}$. In BRAF mutant melanoma patients, MAPK pathway inhibitors increase the number of TAMs and TNF- $\alpha$ expression ${ }^{[50]}$. $\mathrm{BRAF}^{\mathrm{V} 600 \mathrm{E}}$ has also been shown to drive expression of IL-6, IL-10 and VEGF, cytokines that promote a tolerogenic monocyte-derived DC phenotype in vitro, a process that affects anti-tumor $\mathrm{T}$ cell 
function in vivo ${ }^{[51]}$.

The tumor suppressor p53 is the most mutated gene in human cancer ${ }^{[52]}$. Compelling evidence suggests that p53 mutation leads to the gain-of-function or activation of non-autonomous pathways, which either directly or indirectly promote tumorigenesis ${ }^{[53]}$. P53 dysfunction in various cellular compartments of the tumor immune microenvironment leads to immune suppression and immune evasion ${ }^{[52]}$.

Multiple reports have showed that the oncogene $\mathrm{KRAS}^{\mathrm{G} 12 \mathrm{D}}$ can drive pancreatic ductal adenocarcinoma cells (PDAC) to secrete high levels of the growth factor GM-CSF, which is associated with an increase in tumor-associated $\mathrm{Grl}^{+} \mathrm{CD} 1 \mathrm{lb}^{+}$myeloid cells with reported immunosuppressive function ${ }^{[54]}$. These data show that oncogenes promote the establishment of an immunosuppressive TIME, which is likely to support malignant development. Therefore the systematic analysis of the correlation between cancer genome and the TIME will generate new perspectives on the comprehension of the carcinogenesis and cancer treatment.

\section{CHARACTERS OF THE TIME DURING TUMOR METASTASIS}

Tumor metastasis is responsible for more than $90 \%$ of cancer mortality, yet still remains the least understood stage of tumor development. It has been proven that primary tumors can secrete cytokines, growth factors and proteases that mediate the establishment of the specific TIME in distant organs that are sites of future metastasis, in terms of the formation of the "pre-metastatic niche" ${ }^{\text {[55] }}$.

Epithelial-to-mesenchymal transition (EMT) of tumor cells is associated with distant metastasis. During EMT, the tumor cells undergo morphological changes that confer enhanced motility and reduced intercellular adhesion. It is reported that tumor recruitment of MDSC promotes EMT ${ }^{[56]}$. Mast cells in the breast tumor immune microenvironment can secrete tryptase, which results in enhanced rates of metastasis to the lymph nodes and high-grade breast tumors ${ }^{[57]}$. In the polysome middle T antigen (PyMT) model of breast cancer, studies showed that macrophages exposed to IL-4 are able to upregulate the expression of cysteine protease cathepsin $\mathrm{B}$ and lead to increased lung metastases ${ }^{[58]}$. In the colorectal cancer model, immature $\mathrm{CCRl}^{+}$myeloid cells recruited from bone marrow migrate toward the CCR1 ligand (CCL9) expressed in the tumor epithelium and also promote tumor invasion ${ }^{[59]}$. Immunosuppressive $\mathrm{CD} 4^{+} \mathrm{T}$ cells recruited to the TIME can secrete IL-4 and IL-13 to convert tumor suppressive Ml macrophages to a tumor-promoting M2 phenotype, which have been shown to support breast cancer metastasis to the lung ${ }^{[00]}$. In particular, breast cancer cells are able to recruit tumor-associated macrophages through the production of CSFl and CXCL12. Lewis lung carcinoma (LLC) cells can activate macrophages by the secretion of TNF- $\alpha$ and IL- 6 , which induce invasion and metastasis by signaling to toll-like receptor 2 (TLR2) and toll-like receptor 6 (TLR6) ${ }^{[61]}$. These data emphasize that the immunosuppressive mediators in the TIME may encourage the tumor metastasis.

\section{THE TIME AS A TARGET FOR IMMU- NOTHERAPY}

Immunotherapy represents the third important historical wave in the systemic treatment of cancer after chemotherapy ${ }^{[62]}$. Its first clinical application was in the late 19th century when William B. Coley observed tumor shrinkage (and in some cases disappearance) following the injection of bacterial products in and around tumors.

Immune checkpoint therapies that use blocking antibodies for CTLA-4, and PD-1 have shown impressive efficacy in clinical trials. Cancer Immunotherapy was voted as 2013's Breakthrough of the Year by the journal Science $^{[63]}$. In 2011, the US Foods and Drug Administration (FDA) approved anti-CTLA-4 antibodies (ipilimumab) for use of treating melanoma, which marked the beginning of a new era in cancer immunotherapy ${ }^{[6]]}$. The FDA also approved sipuleucel-T (trade name Provenge ${ }^{\mathbb{R}}$ ) for advanced prostate cancer ${ }^{[65]}$. Leukocyte migration are regulated by chemokines, which raises the idea that manipulating the TIME chemokine profile may recruit sufficient numbers of effector cells into tumor tissues for tumor destruction ${ }^{[66]}$. CXCL10 is a kind of chemokine that recruits $\mathrm{T}$ cells and dipeptidyl peptidase 4 (DPP4) (an enzyme that inactivates CXCL10 in tumor tissues) ${ }^{[67]}$. DPP4 inhibitors can increase the number of tumor-infiltrating $\mathrm{T}$ cells and can also be used in combination with other immunotherapies ${ }^{[68]}$. In contrast to chemokines, cytokines are a broad group of proteins that can modulate immune responses. The fusion protein generated by linking IFN- $\alpha$ to an anti-CD20 antibody can induce tumor regression, since IFN- $\alpha$ plays important roles in anti-tumor responses ${ }^{[69]}$. In another study, the anti-EGFR-IFN- $\alpha$ fusion protein also showed potent anti-tumor activities, such as targeting DCs to increase antigen cross-presentation, leading to increased T-cell priming in the TIME ${ }^{[70]}$. The approach that holds the most clinical promise today is the generation of chimeric antigen receptors (CARs). CARs are fusion proteins which incorporate antigenrecognition domains and T-cell activation domains ${ }^{[7]]}$. 
T cells can be changed to express CARs and transferred to patients. Two CART19 products, CTL019 (Kymriah $^{\circledR}$, Novartis) and KTE-19 (Yescarta ${ }^{\circledR}$, Kite Pharma/ Gilead) have already been approved by the US FDA to treat refractory or relapsed paediatric and young-adult B-ALL or refractory or relapsed adult diffuse largeB cell lymphoma, respectively ${ }^{[72]}$. The development of immunotherapy underscores the importance of understanding basic tumor immunology and the roles of the immunosuppressive TIME, and thus is shaping the direction of current cancer research and having a large influence on cancer therapy.

\section{PERSPECTIVES}

With the rapid development of science and technology, large progress has been made in the tumor immune microenvironment. Increasing evidence suggests that the disruption of TIME may serve as a novel and critical therapeutic paradigm to fight cancer ${ }^{[73]}$. Clinical trials of combination therapy that target both the tumor and TIME are being conducted in several types of advanced cancer ${ }^{[74,75]}$.

To date, there are still challenges in this field: since the different subsets of TIME may lead to different clinical outcomes, further studies will need to be conducted in order to find more functions and mechanisms in the subsets of TIME which can instruct the clinical therapy. Proper in vitro and in vivo models need to be constructed for analyzing the interactions between cancer cells and the immune microenvironment. It is still largely unknown how different immune cells cooperate with each other within tumor tissues ${ }^{[76]}$. Thus, the molecular mechanisms that regulate cell-to-cell interaction need to be uncovered. The composition of the immune cells in the TIME in primary tumors and metastases is highly varied ${ }^{[77]}$, therefore immune therapies that target different kinds of TIME are much required for better efficacy.

\section{Referenes}

[1] Hui L, Chen Y. Tumor microenvironment: Sanctuary of the devil. Cancer Lett, 2015, 368(1):7-13.

[2] Douglas H, Robert AW. Hallmarks of cancer: The next generation. Cell, 2011, 144(5): 646-74.

[3] Aggarwal BB, Shishodia S, Sandur SK, et al. Inflammation and cancer: How hot is the link? Biochem Pharmacol, 2006, 72(11):1605-21.

[4] Palucka AK, Coussens LM. The basis of oncoimmunology. Cell, 2016, 164(6):1233-47.

[5] Helmy KY, Patel SA, Nahas GR, et al. Cancer immunotherapy: accomplishments to date and future promise. Ther Deliv, 2013, 4(10):1307-20.

[6] Topalian SL, Drake CG, Pardoll DM. Immune checkpoint blockade: A common denominator approach to cancer therapy. Cancer Cell, 2015, 27(4): 450-61.

[7] Spranger S. Mechanisms of tumor escape in the context of the T-cell-inflamed and the non-T-cell-inflamed tumor microenvironment. Int Immunol, 2016, 28(8):91-383.

[8] Ademmer K, Ebert M, Muller-Ostermeyer F, et al. Effector $\mathrm{T}$ lymphocyte subsets in human pancreatic cancer: detection of $\mathrm{CD}^{+} \mathrm{CD} 18^{+}$cells and $\mathrm{CD}^{+} \mathrm{CD} 103^{+}$cells by multi-epitope imaging. Clin Exp Immunol, 1998, 112: 21-6.

[9] Spranger S, Spaapen RM, Zha Y, et al. Up-regulation of PD-L1, IDO, and T(regs) in the melanoma tumor microenvironment is driven by CD8(+) T cells. Sci Transl Med, 2013, 5(200):116-200.

[10] Tumeh PC, Harview CL, Yearley JH, et al. PD-1 blockade induces responses by inhibiting adaptive immune resistance. Nature, 2014, 515(7528):568-71.

[11] DuPage M, Cheung AF, Mazumdar C, et al. Endogenous $\mathrm{T}$ cell responses to antigens expressed in lung adenocarcinomas delay malignant tumor progression. Cancer Cell, 2011, 19(1):72-85.

[12] Binnewies M, Roberts EW, Kersten K, et al. Understanding the tumor immune microenvironment (TIME) for effective therapy. Nat Med, 2018, 24(5):541-50.

[13] Lutz ER, Wu AA, Bigelow E, et al. Immunotherapy converts nonimmunogenic pancreatic tumors into immunogenic foci of immune regulation. Cancer Immunol Res, 2014, 2(7): 616-31.

[14] Lee HJ, Park IA, Song IH, et al. Tertiary lymphoid structures: prognostic significance and relationship with tumour-infiltrating lymphocytes in triple-negative breast cancer. J Clin Pathol, 2016, 69(5): 422-30.

[15] Gajewski TF, Meng Y, Blank C, et al. Immune resistance orchestrated by the tumor microenvironment. Immunol Rev, 2006, 213:131-76.

[16] Elinav E, Nowarski R, Thaiss CA, et al. Inflammationinduced cancer: crosstalk between tumours, immune cells and microorganisms. Nat Rev Cancer, 2013, 13(11):75971.

[17] Gajewski TF, Schreiber H, Fu YX. Innate and adaptive immune cells in the tumor microenvironment. Nat Immunol, 2013, 14(10):1014-36.

[18] Francisco LM, Salinas VH, Brown KE, et al. PD-L1 regulates the development, maintenance, and function of induced regulatory T cells. J Exp Med, 2009, 206(13):301529.

[19] Fridman WH, Pages F, Sautes-Fridman C, et al. The immune contexture in human tumours: impact on clinical outcome. Nat Rev Cancer, 2012, 12(4):298-306.

[20] Wargo JA, Reddy SM, Reuben A, et al. Monitoring immune responses in the tumor microenvironment. Curr Opin Immunol, 2016, 4:23-31.

[21] Chang SH, Mirabolfathinejad SG, Katta H, et al. T helper 17 cells play a critical pathogenic role in lung cancer. Proc Natl Acad Sci U S A, 2014, 111(15):5664-73.

[22] Pastille E, Bardini K, Fleissner D, et al. Transient ablation of regulatory $\mathrm{T}$ cells improves antitumor immunity in co- 
litis-associated colon cancer. Cancer Res, 2014, 74(16): 4258-327.

[23] Bauer CA, Kim EY, Marangoni F, et al. Dynamic Treg interactions with intratumoral APCs promote local CTL dysfunction. J Clin Invest, 2014, 124(6):2425-65.

[24] Lim HW, Hillsamer P, Banham AH, et al. Cutting edge: direct suppression of $\mathrm{B}$ cells by $\mathrm{CD} 4^{+} \mathrm{CD} 25^{+}$regulatory $\mathrm{T}$ cells. J Immunol, 2005, 175(7):4180-3.

[25] Kumar V. The nature of myeloid-derived suppressor cells in the tumor microenvironment. Trends Immunol, 2016, 37(3):208-20.

[26] Marvel D, Gabrilovich DI. Myeloid-derived suppressor cells in the tumor microenvironment: expect the unexpected. J Clin Invest, 2015, 125(9):3356-420.

[27] Yang L, DeBusk LM, Fukuda K, et al. Expansion of myeloid immune suppressor $\mathrm{Gr}^{+} \mathrm{CD} 1 \mathrm{lb}^{+}$cells in tumorbearing host directly promotes tumor angiogenesis. Cancer Cell, 2004, 6(4): 409-30.

[28] Komohara YM, Takeya M. Clinical significance of macrophage heterogeneity in human malignant tumors. Cancer Sci, 2014,105(1):1-8.

[29] Ostrand-Rosenberg S, Sinha P, Beury DW, et al. Crosstalk between myeloid-derived suppressor cells (MDSC), macrophages, and dendritic cells enhances tumorinduced immune suppression. Semin Cancer Biol, 2012, 22(4):275-356.

[30] David E. Tumour immunogenicity, antigen presentation and immunological barriers in cancer immunotherapy. New J Sci, 2014, 2014:734515.

[31] Gabrilovich DI, Ostrand-Rosenberg S, Bronte V. Coordinated regulation of myeloid cells by tumours. Nat Rev Immunol, 2012, 12(4):253-321.

[32] Strioga M. Dendritic cells and their role in tumor immunosurveillance. Innate Immun, 2013, 19(1):98-111.

[33] Seton-Rogers S. Tumour immunology: dendritic cell switch. Nat Rev Cancer, 2012,12(4): 230.

[34] Shelburne CP, Nakano H, St John AL, et al. Mast cells augment adaptive immunity by orchestrating dendritic cell trafficking through infected tissues. Cell Host Microbe, 2009, 6(4):331-73.

[35] Braster R, Bogels M, Beelen RHJ, et al. The delicate balance of macrophages in colorectal cancer; their role in tumour development and therapeutic potential. Immunobiology, 2017, 222(1):21-30.

[36] Haanen JB, Robert C. Immune checkpoint inhibitors. Prog Tumor Res, 2015, 42: 55-66.

[37] Pardoll DM. The blockade of immune checkpoints in cancer immunotherapy. Nat Rev Cancer, 2012, 12(4):252316.

[38] Hamanishi J. PD-1/PD-L1 blockade in cancer treatment: perspectives and issues. Int J Clin Oncol, 2016, 21(3):462-73.

[39] Brunet JF. A new member of the immunoglobulin superfamily--CTLA-4. Nature, 1987, 328(6127):267-337.

[40] He Y, Rivard CJ, Rozeboom L, et al. Lymphocyteactivation gene-3, an important immune checkpoint in cancer. Cancer Sci, 2016, 107(9):1193-200.
[41] Torphy RJ, Schulick RD, Zhu Y. Newly emerging immune checkpoints: Promises for future cancer therapy. Int J Mol Sci, 2017,18(12): E2642.

[42] Xu F, Jin TQ, Zhu YW, et al. Immune checkpoint therapy in liver cancer. J Exp Clin Cancer Res, 2018, 37(1):110.

[43] Li J, Jie HB, Lei Y, et al. PD-1/SHP-2 inhibits Tc1/Thl phenotypic responses and the activation of $\mathrm{T}$ cells in the tumor microenvironment. Cancer Res, 2015, 75(3): 50818.

[44] Patsoukis N, Sari D, Boussiotis VA. PD-1 inhibits T cell proliferation by upregulating $\mathrm{p} 27$ and $\mathrm{p} 15$ and suppressing Cdc25A. Cell Cycle, 2012, 11(23):4305-14.

[45] Duraiswamy J, Freeman GJ, Coukos G. Therapeutic PD-1 pathway blockade augments with other modalities of immunotherapy T-cell function to prevent immune decline in ovarian cancer. Cancer Res, 2013, 73(23):6900-12.

[46] Sedy JR, Gavrieli M, Potter KG, et al. B and T lymphocyte attenuator regulates $\mathrm{T}$ cell activation through interaction with herpesvirus entry mediator. Nat Immunol, 2005, 6(1):90-8.

[47] Monney L, Sabatos CA, Gaglia JL, et al. Th1-specific cell surface protein Tim-3 regulates macrophage activation and severity of an autoimmune disease. Nature, 2002, 415(6871): 536-41.

[48] Hiroyuki Y, Kohzoh I. Microsatellite instability: an update. Arch Toxicol, 2015, 89(6): 899-921.

[49] Llosa NJ, Cruise M, Tam A, et al. The vigorous immune microenvironment of microsatellite instable colon cancer is balanced by multiple counter-inhibitory checkpoints. Cancer Discov, 2015, 5(1): 43-51.

[50] Katerinaki E, Evans GS, Lorigan PC, et al. TNF-alpha increases human melanoma cell invasion and migration in vitro: the role of proteolytic enzymes. Br J Cancer, 2003, 89(6):1123-9.

[51] Smith MP, Sanchez-Laorden B, O’Brien K, et al. The immune microenvironment confers resistance to MAPK pathway inhibitors through macrophage-derived TNFalpha. Cancer Discov, 2014, 4(10):1214-29.

[52] Meek DW. Regulation of the p53 response and its relationship to cancer. Biochem J, 2015, 469(3):325-71.

[53] Cui Y, Guo G. Immunomodulatory function of the tumor suppressor p53 in host immune response and the tumor microenvironment. Int J Mol Sci, 2016, 17(11):1942.

[54] Pylayeva-Gupta Y, Lee KE, Hajdu CH, et al. Oncogenic Kras-induced GM-CSF production promotes the development of pancreatic neoplasia. Cancer Cell, 2012, 21(6):836-83.

[55] Langley RR, Fidler IJ. The seed and soil hypothesis revisited-The role of tumor-stroma interactions in metastasis to different organs. Int J Cancer, 2011, 128(11):2527-35.

[56] Toh B, Wang X, Keeble J, et al. Mesenchymal transition and dissemination of cancer cells is driven by myeloidderived suppressor cells infiltrating the primary tumor. PLoS Biol, 2011, 9(9): el001162.

[57] Xiang M, Gu YP, Zhao FD, et al. Mast cell tryptase promotes breast cancer migration and invasion. Oncol Rep, 2010, 23(3):615-24. 
[58] Vasiljeva O, Papazoglou A, Peters C, et al. Tumor cellderived and macrophage-derived cathepsin B promotes progression and lung metastasis of mammary cancer. Cancer Res, 2006, 66(10):5242-92.

[59] Itatani Y, Kawada KJ, Fujishita T, et al. Loss of SMAD4 from colorectal cancer cells promotes CCL15 expression to recruit CCR1+ myeloid cells and facilitate liver metastasis. Gastroenterology, 2013, 145(5):1064-75.

[60] Su SC, Liu Q, Chen JQ, et al. A positive feedback loop between mesenchymal-like cancer cells and macrophages is essential to breast cancer metastasis. Cancer Cell, 2014, 25(5):605-25.

[61] Kim S, Takahashi H, Lin WW, et al. Carcinoma-produced factors activate myeloid cells through TLR2 to stimulate metastasis. Nature, 2009, 457(7225):102-8.

[62] Lesterhuis WJ, Haanen JB, Punt CJ. Cancer immunotherapy-revisited. Nat Rev Drug Discov, 2011, 10(8): 591600.

[63] Couzin-Frankel J. Breakthrough of the year 2013: Cancer immunotherapy. Science, 2013, 342(6165):1432-5.

[64] Yang Y. Cancer immunotherapy: harnessing the immune system to battle cancer. J Clin Invest, 2015, 125(9):3335-7.

[65] Singh BH, Gulley JL. Immunotherapy and therapeutic vaccines in prostate cancer: an update on current strategies and clinical implications. Asian J Androl, 2014, 16(3):364-435.

[66] Franciszkiewic K, Boissonnas A, Boutet M, et al. Role of chemokines and chemokine receptors in shaping the effector phase of the antitumor immune response. Cancer Res, 2012, 72(24):6325-32.

[67] Decalf J, Tarbell KV, Casrouge A, et al. Inhibition of DPP4 activity in humans establishes its in vivo role in CXCL10 post-translational modification: prospective placebo-controlled clinical studies. EMBO Mol Med, 2016, 8(6): 679-83.

[68] Barreira da Silva R, Laird ME, Yatim N, et al. Dipepti- dylpeptidase 4 inhibition enhances lymphocyte trafficking, improving both naturally occurring tumor immunity and immunotherapy. Nat Immunol, 2015, 16(8):850-8.

[69] Xuan C, Steward KK, Timmerman JM, et al. Targeted delivery of interferon-alpha via fusion to anti-CD20 results in potent antitumor activity against B-cell lymphoma. Blood, 2010, 115(14):2864-71.

[70] Yang X, Zhang X, Fu ML, et la. Targeting the tumor microenvironment with interferon-b bridges innate and adaptive immune responses. Cancer Cell, 2014, 25(1): 37-48.

[71] Kochenderfer JN, Dudley ME, Kassim SH, et al. Chemotherapy-refractory diffuse large B-cell lymphoma and indolent B-cell malignancies can be effectively treated with autologous $\mathrm{T}$ cells expressing an anti-CD19 chimeric antigen receptor. J Clin Oncol, 2015, 33(6):540-9.

[72] Ascierto PA, Brugarolas J, Buonaguro L, et al. Perspectives in immunotherapy: meeting report from the Immunotherapy Bridge (29-30 November, 2017, Naples, Italy). J Immunother Cancer, 2018, 6(1):69.

[73] Tang H, Qiao J, Fu YX. Immunotherapy and tumor microenvironment. Cancer Lett, 2016, 370(1): 85-90.

[74] Koido S, Ohkusa T, Homma S, et al. Immunotherapy for colorectal cancer. World J Gastroenterol, 2013, 19(46):8531-42.

[75] Simons JW. Prostate cancer immunotherapy: beyond immunity to curability. Cancer Immunol Res, 2014, 2(11):1034-43.

[76] Chew V, Toh HC, Abastado JP. Immune microenvironment in tumor progression: Characteristics and challenges for therapy. J Oncol, 2012, 2012: 608406.

[77] Liu Y, Cao X. Immunosuppressive cells in tumor immune escape and metastasis. J Mol Med (Berl), 2016, 94(5):509-22.

(Received 21 July 2018, Revised 30 August 2018, Accepted 07 September 2018) 